\title{
The Time Course of Responding to Aversiveness in Females with a History of Non-Suicidal Self-Injury
}

\author{
Carter J. Funkhouser ${ }^{a}$, Kelly A. Correa ${ }^{a}$, Vivian L. Carrillo ${ }^{a}$, David M. Klemballa ${ }^{a}$, and \\ Stewart A. Shankman ${ }^{\mathrm{a}, \mathrm{d}, \mathrm{e}}$ \\ aUniversity of Illinois at Chicago, Department of Psychology \\ dUniversity of Illinois at Chicago, Department of Psychiatry \\ eNorthwestern University, Department of Psychiatry and Behavioral Sciences
}

\begin{abstract}
Non-suicidal self-injury is a risk factor for suicidal behavior, particularly in females. Two prominent theories of suicide suggest that habituation to the psychophysiological aversiveness of NSSI is a mechanism by which NSSI exposure may lead to increased risk for suicide. Several laboratory studies examining the relationship between physiological habituation and suicide attempt history have yielded mixed results, potentially due to their use of broad measures of physiological arousal and/or focus on specific psychopathologies. However, no studies have examined the association between the time course (e.g., habituation, initial reactivity) of responding to aversiveness and NSSI, which may help to elucidate psychophysiological mechanisms of NSSI. Therefore, we examined habituation and initial reactivity to aversiveness (indexed by the time course of acoustic startle reflex, a well-validated measure of defensive responding) in three groups of young adult females - those with a history of NSSI, psychiatric controls matched on potential confounds (e.g., psychopathology, trauma history, demographics), and healthy controls. Results indicated that individuals with a history of NSSI exhibited blunted initial reactivity and marginally slower habituation to aversiveness relative to the two control groups. The NSSI group's insensitivity to aversiveness may reflect prior psychophysiological habituation, and may be a mechanism through which prior NSSI exposure leads to increased risk for suicidal behavior.
\end{abstract}

\section{Keywords}

non-suicidal self-injury; habituation; initial reactivity; time course; startle

Correspondence concerning this article should be addressed to Stewart A. Shankman, Department of Psychiatry and Behavioral Sciences, Northwestern University, Chicago, IL, 60611. stew.shankman@northwestern.edu.

Publisher's Disclaimer: This is a PDF file of an unedited manuscript that has been accepted for publication. As a service to our customers we are providing this early version of the manuscript. The manuscript will undergo copyediting, typesetting, and review of the resulting proof before it is published in its final citable form. Please note that during the production process errors may be discovered which could affect the content, and all legal disclaimers that apply to the journal pertain. 


\section{Introduction}

The definition of non-suicidal self-injury (NSSI) varies, but broadly, the behavior refers to the direct, deliberate destruction of one's body tissue in the absence of lethal intention (Nock, 2009). Common methods of NSSI include cutting, burning, scratching, biting, hitting, and skin picking (e.g., Klonsky, 2011). NSSI typically begins during early adolescence and it is estimated that 13-23\% of adolescents have engaged in NSSI (Jacobson \& Gould, 2007). Although there are multiple reasons why people engage in NSSI, most models of NSSI posit that in many cases, it serves to reduce aversive emotions such as sadness and anxiety (Klonsky, 2007; Nock \& Prinstein, 2004).

NSSI has consistently been shown to be a risk factor for suicidal behavior. Nock, Joiner, Gordon, Lloyd-Richardson, and Prinstein (2006) found that $70 \%$ of adolescents who had engaged in NSSI had attempted suicide at least once, and 55\% reported multiple attempts. Furthermore, longitudinal studies have shown NSSI is a prospective and unidirectional predictor of suicidal behavior. These studies indicate that individuals with a history of NSSI are more than five times more likely to experience suicidal ideation and 3-15 times more likely to attempt suicide over follow-up periods ranging from 24 weeks to 3 years (Asarnow et al., 2011; Guan et al., 2012; Prinstein et al., 2008; Whitlock et al., 2013; Wilkinson et al., 2011). Importantly, both Asarnow et al. (2011) and Wilkinson et al. (2011) reported that NSSI history predicted whether participants would later attempt suicide, even after controlling for prior suicide attempts. Additionally, females have higher rates of NSSI and suicidal behavior (Asarnow et al., 2011; Prinstein et al., 2008; Wilkinson et al., 2011) and more severe levels of NSSI correlates (e.g., psychopathology; Victor et al., 2018), suggesting that females may be especially vulnerable to NSSI and suicidal behavior. These findings highlight the importance of NSSI as an indicator of risk for future suicide attempts.

However, the mechanism underlying the association between NSSI and suicidal behavior is unclear. Two prominent theories of suicide propose that physiological habituation to aversiveness may be a mechanism by which NSSI leads to suicidal behavior. Gateway theory suggests that there is a continuum of self-injurious behaviors with NSSI at one extreme and completed suicide at the other and NSSI serves as a "gateway" to more severe self-harm behaviors (e.g., attempted suicide) by reducing inhibition through habituation to self-harm (Whitlock et al., 2013). Similarly, the Interpersonal-Psychological Theory of Suicide (IPTS) suggests that repeated exposure to painful or provocative experiences such as NSSI leads to "acquired capability" for suicide (Joiner, 2005). Although the two theories differ in some ways (e.g., gateway theory suggests only behaviors with similar experiential qualities [i.e., NSSI] can lead to suicidal behavior, whereas acquired capability theory suggests that NSSI is one of many painful or provocative experiences that can lead to acquired capability for suicide; Smith \& Cukrowicz, 2010; Whitlock et al., 2013), both theories suggest that habituation to aversiveness plays an important role in suicidal behavior.

Several studies have attempted to test this hypothesis by examining physiological habituation in suicide attempters. Electrodermal studies have found faster habituation in suicide attempters and those who later die by suicide compared to suicide ideators and nonsuicidal depressed participants (Edman, Asberg, Levander, \& Schalling, 1986; Jandl, Steyer, 
\& Kaschka, 2010; Keller, Wolfersdorf, Straub, \& Hole, 1991; Thorell et al., 2013; Wolfersdorf et al., 1999). However, other studies have failed to find an association. Smith et al. (2015) examined the relationship between physiological habituation of the acoustic startle reflex, suicide attempt history, and acquired capability, and found no relationship between physiological habituation and self-reported acquired capability for suicide or suicide attempt history. Although research on the time course of defensive responding has focused on physiological habituation of defensive responding (Klumpp \& Shankman, 2018), abnormal initial reactivity to painful or aversive stimuli may also relate to NSSI. Initial reactivity (i.e., the beginning intercept of an individual's responding over time) is thought to reflect initial stimulus processing, whereas habituation (i.e., the slope of responding over time) may reflect one's ability to inhibit reactions to the stimuli over time (Banks et al., 2007; Phan et al., 2005). Although not examined in the laboratory studies discussed above, several studies have examined average (not initial) reactivity to emotional stimuli in NSSI with mixed results. For example, Glenn, Blumenthal, Klonsky, \& Hajcak (2011) examined startle reactivity to emotional images and found no differences between individuals with a history of NSSI and controls, but Nock and Mendes (2008) found NSSI was associated with physiological hyperarousal (i.e., increased skin conductance) to psychological distress. However, these studies did not examine the time course (e.g., habituation, initial reactivity) of responding, which may help to elucidate the role of physiological reactivity in NSSI.

These studies had several limitations that prevent them from being definitive tests of the role of habituation to aversiveness in NSSI. First, many of these studies only included participants who were currently depressed. Although some individuals who engage in selfinjurious behaviors are depressed, NSSI, suicidal behavior, and the time course of responding to aversiveness (e.g., habituation) are transdiagnostic (Fleischmann, Bertolote, Belfer, \& Beutrais, 2005; Nock et al., 2006). Therefore, it is important to examine the time course of responding to aversiveness independent of diagnosis, an aim in line with the Research Domain Criteria (RDoC) initiative (Westlund Schreiner, Klimes-Dougan, Begnel, $\&$ Cullen, 2015). Second, prior studies focused on habituation as a function of suicide attempt history and not NSSI history, which is important because there are potentially different paths that lead to attempted suicide, some of which may not involve prior suicidal behavior (and perhaps not acquired capability either; Brackman et al., 2016; Hamza et al., 2012). To our knowledge, no laboratory studies have investigated the time course of psychophysiological responding in individuals specifically with a history of NSSI. Third, many of these studies used measures that are not valence specific but rather reflect overall emotional arousal (e.g., electrodermal activity). The acoustic startle reflex, on the other hand, is a basic, cross-species reflex that reflects defensive motivation and is sensitive to the valence of an individual's internal state (Lang, 1995), and therefore may be a more relevant measure of psychophysiological responding to aversiveness in NSSI.

Although gateway theory and the IPTS propose that physiological habituation to aversiveness is a critical component leading to suicidal behavior, there are several third variables that may entirely explain the association between NSSI and suicidal behavior. For example, as suicide completers and those who engage in NSSI have comparable rates of psychopathology (88.6\% vs. $87.6 \%$, Fleischmann et al., 2005; Nock et al., 2006), it is possible that having a mental disorder increases risk for both NSSI and suicidal behavior. No 
study on the time course of psychophysiological responding in NSSI has attempted to rule out these potential confounds. Indeed, many (if not most) studies in the broader NSSI literature either compare individuals with NSSI to healthy controls or, as mentioned above, focus on NSSI exclusively in individuals with certain psychopathologies such as depression (e.g., Asarnow et al., 2011; Wilkinson et al., 2011), neither of which allows researchers to separate the effects of NSSI from confounding psychopathology. Some studies (e.g., Andover \& Gibb, 2010) have sought to address this problem by statistically controlling for potential demographic or psychopathological third variables, but this may also be problematic because NSSI is so closely associated with psychopathology that statistically controlling for psychopathology may remove key variance associated with the dependent variable of interest (Miller \& Chapman, 2001). Therefore, one goal of this study is to select a control group that is matched to the NSSI group on potential confounds, such as psychopathology and key demographics.

To test these aims, the present study conducted secondary analyses from a larger psychophysiological project (Gorka et al., 2016; Shankman et al., 2018, Weinberg et al., 2015) to examine the time course of startle responding in three groups of individuals - (a) those with a history of NSSI, (b) psychiatric controls matched on demographics and psychopathology, and (c) healthy controls. While startle studies often examine how the startle response is modulated by context, other studies have examined responses to unmodulated acoustic startle probes as indicators of defensive responding (e.g., Miller, Curtin, \& Patrick, 1999) including studies on the time course of defensive responding (Blanch, Balada, \& Aluja, 2014; Campbell et al., 2014; Smith et al., 2015). Although comparing individuals with NSSI to matched psychiatric controls served to address the potential effects of third variables and isolate the effects of NSSI, we included the group of healthy controls to determine whether individuals with a history of NSSI exhibited abnormal (and not just different from psychiatric controls) habituation or initial reactivity.

Additionally, because this investigation was a secondary analysis of data from a larger study not specifically designed to examine NSSI, we sought to validate our assessment of NSSI by examining group differences in pain threshold (i.e., the lowest level of stimulus intensity that is perceived as painful) as individuals who have engaged in have consistently exhibited greater pain thresholds (Koenig, Thayer, \& Kaess, 2016). Because both gateway theory and the IPTS suggest that NSSI habituates individuals to aversiveness, we hypothesized that individuals with a history of NSSI would exhibit blunted psychophysiological initial reactivity and faster habituation relative to the psychiatric controls and healthy controls.

\section{Methods}

\subsection{Participants}

Ninety female participants that were 18 to 29 years old $(M=21.73, S D=2.81)$ were drawn from a larger, NIMH-funded, family study (see Gorka et al., 2016 and Shankman et al., 2018 for additional details). Advertisements (fliers, internet postings, etc.) were used to recruit participants from the community and from mental health clinics. A Research Domain Criteria (RDoC) approach was taken to participant recruitment such that recruitment screening was agnostic to Diagnostic and Statistical Manual of Mental Disorders (DSM) 
diagnostic categories (beyond the exclusion criteria listed below). However, participants with severe internalizing psychopathology were oversampled to ensure that the sample was clinically relevant. Specifically, the Depression, Anxiety, and Stress Scale (DASS; Lovibond $\&$ Lovibond, 1995) was administered during the initial phone screen to ensure that the severity of internalizing symptomology within the sample was normally distributed, but also had a higher average general psychological distress score $(M=10.35, S D=10.07)$ than the general population $(M=8.30, S D=9.83$; Crawford, Cayley, Lovibond, Wilson, \& Hartley, 2011). Consequently, the sample included a wide range of psychopathologies, as well as healthy controls.

Inclusion criteria specified that participants had at least one full biological sibling that was also willing to participate in the study. While both siblings participated in the larger study, the sample for the present study only included one sibling per family (so as not to violate the statistical assumption of independence of observations). Exclusion criteria included personal or family history of psychosis or mania at the time of the interview (given that psychosis and mania have been shown to be separable from internalizing and externalizing disorders; Caspi et al., 2014; Kotov et al., 2011; Krueger et al., 1998; Markon, 2010), inability to read or write in English, history of serious head trauma, and left-handedness (to protect against confounds with the neurophysiological data collected for the main aims of the larger study).

We extracted groups of participants with a history of NSSI ( $n=16)$, psychiatric controls ( $n=$ 28 ), and healthy controls $(n=43)$ that were matched on age, ethnicity, and years of education. As $96 \%$ of participants in the larger study who endorsed a history of NSSI were female and previous studies have demonstrated gender differences in rates and methods of NSSI as well as suicide attempts (Andover, Primack, Gibb, \& Pepper, 2010; Bresin \& Schoenleber, 2015; Sornberger, Heath, Toste, \& McLouth, 2012; Victor \& Klonsky, 2014), only females were included in the extracted groups. In order to test for the specificity of the effects of NSSI history on responses to aversiveness, a psychiatric control group was also extracted that was comparable to the NSSI group in rates of suicide attempt, lifetime and current psychopathology (e.g., depression, anxiety disorders, substance use disorders), childhood abuse and neglect, psychiatric medication use, and global symptom severity and impairment as these variables are likely to be confounded with NSSI (Andover \& Gibb, 2010; Ballard et al., 2014; Gorka, Liu, Sarapas, \& Shankman, 2015; Nock et al., 2006; Glassman, Weierich, Hooley, Deliberto, \& Nock, 2007; Jovanovic et al., 2010; Fournier et al., 2010; Rodriguez-Fornells, Riba, Gironell, Kulisevksy, \& Barbanoj, 1999; Glenn \& Klonsky, 2013; Muehlenkamp, \& Gutierrez, 2007). There were no sibling pairs for which both siblings had a history of NSSI. For sibling pairs in which one sibling had a history of NSSI and the other met criteria for one of the two control groups, the sibling with a NSSI history was selected for the NSSI group and the other sibling was excluded. In cases in which both siblings met criteria for either of the control groups, one of the siblings was randomly selected to be included and the other sibling was excluded. Refer to Table 1 for participant demographics and clinical characteristics. 


\subsection{Measures}

\subsubsection{Structured Clinical Interview for Diagnostic and Statistical Manual of} Mental Disorders-5 (SCID)—NSSI history and suicide attempt history were assessed using an adapted version of the SCID interview (First, Williams, Karg, \& Spitzer, 2015; Shankman et al., 2018). The NSSI group was defined as those who endorsed a single yes/no item added to the Mood Disorders module that probed whether the individual had ever engaged in NSSI in their lifetime. Two additional items were included to assess the presence (yes/no) and frequency of suicide attempts, respectively. The SCID also assessed lifetime and current psychopathology (i.e., Major Depressive Disorder, Alcohol Use Disorder, Substance Use Disorder, Posttraumatic Stress Disorder, Panic Disorder, Agoraphobia, Social Anxiety Disorder, Specific Phobia, Obsessive Compulsive Disorder, Generalized Anxiety Disorder, Anorexia, Bulimia, and Binge Eating Disorder). Healthy controls were required to have no lifetime diagnosis of any of these disorders. As personal history of psychosis or mania was an exclusion criterion, lifetime psychosis and mania were also assessed using the psychotic screening module and manic episode section from the SCID's Mood Disorders module. Doctoral students and bachelor's level research assistants were trained to criterion on the SCID and were supervised by a licensed clinical psychologist. Interrater agreement was in the fair to substantial ranges for lifetime diagnoses $(\mathrm{k}$ 's $=.46-.87)$ and in the fair to moderate ranges for current diagnoses ( $\mathrm{k}$ 's $=.54-.74)$ with the exception of lifetime $(\mathrm{k}=$. $18)$ and current $(\mathrm{k}=$.29) Social Anxiety Disorder (Shankman et al., 2018; Shrout, 1998).

\subsubsection{Global Assessment of Functioning and Social and Occupational} Functioning Assessment Scale-The Global Assessment of Functioning (GAF; American Psychiatric Association, 1994) was modified slightly to only focus on overall current symptom severity, and the Social and Occupational Functioning Assessment Scale (SOFAS; Goldman, Skodol, \& Lave, 1992) provided a measure of overall functional impairment due to current psychopathology. Both scales are rated from 0 to 100, with lower scores indicating greater levels of symptom severity or impairment.

\subsubsection{Childhood Trauma Questionnaire-Short Form (CTQ-SF)-The CTQ-SF}

(Bernstein et al., 2003) is a self-administered, psychometrically validated questionnaire consisting of 25 items assessing history of childhood abuse and neglect. The CTQ-SF measures five different types of abuse and neglect: emotional abuse, emotional neglect, physical abuse, physical neglect, and sexual abuse. Items are rated based on a 5-point Likert scale ranging from 1 (never true) to 5 (very often true). Cronbach's alphas for the five subscales ranged from .55 to .94 in the present study.

\subsection{Electromyography Startle Habituation Task}

Electromyography (EMG) data were acquired using BioSemi Active Two (Amsterdam, Netherlands). Startle tones were presented using PSYLAB (Contact Precision Instruments; London, UK) and physiological data was recorded using a PC-based acquisition system (Neuroscan 4.3). Startle response was operationalized as the eyeblink response to six $40 \mathrm{~ms}$, $103 \mathrm{db}$ bursts of white noise with near-instantaneous rise time presented binaurally through headphones. The startle eyeblink in both samples was recorded using two $4 \mathrm{~mm} \mathrm{Ag} / \mathrm{AgCl}$ 
electrodes placed over the orbicularis oculi muscle underneath the right eye. Data were collected using a bandpass filter of DC-500-Hz at a sampling rate of $2000-\mathrm{Hz}$.

Following electrode placement, all participants sat in a sound-attenuated, electrically shielded booth approximately 3.5-feet from a 19-inch computer monitor. During each of two 2.5-minute habituation periods, participants were told to relax and focus on a fixation cross on a monitor approximately one meter in front of them while 6 acoustic startle probes were delivered through headphones. Interstimulus intervals (ISIs) between startle probes ranged between 15 and 20 seconds ( $M=17.60$ seconds). In between the first and second habituation periods, shock electrodes were placed on participants' left wrist. Participants were explicitly told that no shocks would be administered during the habituation periods. Although these two habituation periods were intended to prevent early exaggerated startle responding during a subsequent threat-of-shock task that was administered as part of the larger psychophysiological study (Gorka et al., 2013, 2015; Shankman et al., 2013), they are nearly identical to a startle habituation paradigm used in a previous study examining the relationship between suicide attempt history and habituation to aversiveness (Smith et al., 2015).

\subsection{Shock Workup Procedure - Pain Threshold}

After the two startle habituation periods, participants completed a shock work-up procedure in which they received increasing intense shocks beginning at $0.6 \mathrm{~mA}$ and increasing by 0.6 $\mathrm{mA}$ until they reached an intensity level that they described as feeling "highly annoying but not painful." The shock level described as "highly annoying but not painful" served as the measure of pain threshold. Although the idiographic maximum shock levels obtained from this task primarily served to ensure equality in perceived shock aversiveness (Rollman \& Harris, 1987) in the subsequent threat-of-shock task, this shock-work up procedure was nearly identical to paradigms used in previous studies examining pain threshold in the context of NSSI research (Brackman et al., 2016; Franklin, Lee, Hanna, \& Prinstein, 2013). The maximum shock level a participant could receive was $5 \mathrm{~mA}$ and the mean pain threshold was $1.19 \mathrm{~mA}(S D=.50)$.

\subsection{EMG Startle Processing}

Startle blinks were scored according to published guidelines (Blumenthal et al., 2005). Data processing included applying a $28 \mathrm{~Hz}$ high-pass filter, rectifying, then smoothing using a $40 \mathrm{~Hz}$ low-pass filter. Blink response was defined as the peak amplitude of EMG activity within the 20-150-ms period following startle probe onset relative to baseline (average baseline EMG level for the 50-ms preceding the startle probe onset). Each peak was identified by software (BrainVision Analyzer 2.1.0 [Brain Products, Munich, Germany]) but examined by hand to ensure acceptability (e.g., not a double blink). Blinks were scored as non-responses if EMG activity during the $20-150-\mathrm{ms}$ post-stimulus timeframe did not produce a blink peak that was visually differentiated from baseline activity. Blinks were considered to be visually differentiated from baseline activity if the magnitude of the blink was at least two times the mean of the baseline. All blinks were visually evaluated using the same scale to ensure within-subject consistency in the identification of non-responses. Blinks were scored as missing if the baseline period was contaminated with noise, 
movement artifact, or if a spontaneous or voluntary blink began before minimal onset latency and thus interfered with the startle probe-elicited blink response. These definitions are in accordance with established guidelines (Blumenthal et al., 2005). Blink magnitude values (including nonresponse values) were used in analyses.

\subsection{Statistical Analyses}

A complex contrast was utilized to test the hypothesis that pain thresholds (i.e., participants' chosen shock levels) would be higher in the NSSI group compared to controls (i.e., psychiatric and healthy controls combined). Longitudinal mixed linear models were conducted to examine group differences in the within-individual slope and initial reactivity (i.e., the intercept of each individual's slope as estimated by the model) of startle responses over time. Time was coded as the number of elapsed seconds since the administration of the first startle probe, and the slope of startle reactivity was defined as the estimated withinperson slope of startle responses across time. The intercept represented the estimated magnitude of the participant's first startle response (i.e., at time $=0$ ). This operationalization of initial reactivity uses all of the participant's data to estimate the slope intercept or 'initial starting point' and thus is more reliable than using the first startle response (i.e., blink 1 of the habituation period). Missing data (i.e., unscorable blinks) were handled by weighting slope estimates by the number of observations. The statistical significance of fixed effects (e.g., group) was evaluated using $F$ tests, and follow-up $t$-tests were used to test pairwise group differences. In line with recommendations from a recent simulation study (Luke, 2017), degrees of freedom were estimated using Satterthwaite's approximation. Partial Rsquared and the associated 95\% confidence interval (CI) was estimated for each fixed effect using the Kenward-Roger method. All analyses were conducted in version 3.4.3 of R ( R Core Team, 2017) utilizing version 1.1-13 of the lme4 package (Bates, Mächler, Bolker, \& Walker, 2015), version 0.18-0 of the afex package (Singmann, Bolker, Westfall, \& Aust, 2017), version 2.0-33 of the lmerTest package (Kuznetsova, Brockhoff, \& Christensen, 2017), and version 0.1.2 of the r2glmm package (Jaeger, 2017).

\section{Results}

\subsection{Pain Threshold}

As hypothesized, the contrast testing whether the NSSI group $(M=73.47, S D=20.31)$ had a higher pain threshold than the combined control groups $(M=58.00, S D=25.67)$ was significant, $t(81)=2.17, p=.033, d=.48$.

\subsection{Startle Responding}

There were no group differences in the number of non-responses, $F(2,84)=.93, p=.399$, $\eta_{\mathrm{p}}{ }^{2}=.02$, or missing (i.e., unscorable) startle responses, $F(2,84)=.69, p=.505, \eta_{\mathrm{p}}{ }^{2}=.02$, suggesting that any group differences in the time course of startle responding were not due to group differences in nonresponses or missing blinks. A longitudinal mixed linear model was conducted to examine the effects of group, habituation period (with vs. without shock electrodes), time (within the habituation period), and their interactions on startle magnitude. Results revealed significant main effects of group, $F(2,108.40)=4.22, p=.017$, partial $\mathrm{R}^{2}$ $=.08(95 \% \mathrm{CI}[.01, .20])$, and time, $F(1,110.85)=52.46, p<.001$, partial $\mathrm{R}^{2}=.34(95 \% \mathrm{CI}$ 
$[.20, .47]$ ), as well as a marginally significant interaction between group and time (see Figure 1), $F(2,110.61)=2.74, p=.069$, partial $\mathrm{R}^{2}=.05$ (95\% CI $\left.[.01, .17]\right)$. The main effect of habituation period was marginally significant, $F(1,269.39)=3.82, p=.051$, partial $\mathrm{R}^{2}=.01(95 \%$ CI $[.00, .05])$, such that startle magnitudes were marginally greater in the first habituation period compared to the second, but the group by habituation period interaction, $F(2,269.24)<.01, p=.997$, partial $\mathrm{R}^{2}<.01$ (95\% CI [.00, .03]), and group by habituation period by time interaction, $F(2,723.91)=.52, p=.595$, partial $\mathrm{R}^{2}<.01(95 \% \mathrm{CI}[.00, .01])$, were not significant. Thus, the placement of shock electrodes did not impact group differences in the time course of startle responding.

Even though the omnibus group by time interaction only approached significance, due to our a priori hypothesis, pairwise group comparisons were conducted as follow-up analyses. As hypothesized, individuals with a history of NSSI displayed a diminished initial startle response compared to both healthy controls, $b=-31.01, t(126.28)=2.45, p=.016$, and psychiatric controls, $b=-27.87, t(125.73)=2.06, p=.041$. Initial reactivity did not significantly differ between psychiatric controls and healthy controls, $b=-3.14, t(120.55)$ $=.30, p=.762$.

The negative slopes for the psychiatric controls, $b=-.35, t(336.82)=4.59, p<.001$, and healthy controls, $b=-.33, t(340.24)=5.32, p<.001$, significantly differed from zero, indicating that the two control groups habituated to the startle probes over the duration of the habituation periods. The slope of defensive responding in the NSSI group was not different from zero, $b=-.11, t(349.40)=1.03, p=.303$. Contrary to expectations, the slope of startle responding differed among groups at a trend level such that the healthy control group, $b=-$. $22, t(347.03)=1.80, p=.073$, and the psychiatric control group, $b=-.24, t(345.05)=1.84$, $p=.066$, habituated marginally more rapidly than the NSSI group. Slopes of startle magnitude over time did not differ between the psychiatric controls and healthy controls, $b$ $=.02, t(338.18)=.20, p=.838$, indicating that these groups habituated at similar rates. While the NSSI group displayed a diminished initial startle response and habituation rate that did not significantly differ from zero, the NSSI group's startle response to the final startle probe did not differ from that of the healthy controls, $b=-11.45, t(126.83)=.91, p$ $=.366$, or psychiatric controls, $b=-6.54, t(125.55)=.49, p=.628$, suggesting that the NSSI group's final startle response was comparable to that of the two control groups.

\section{Discussion}

This study examined the relationship between NSSI history and the time course of defensive responding to aversiveness in secondary analyses of data from a larger psychophysiological study. As hypothesized, we found that individuals with a history of NSSI exhibited blunted initial reactivity to aversiveness relative to healthy and psychiatric controls. Additionally, habituation rates were marginally greater for the two control groups relative to the NSSI group, who did not significantly habituate. However, group differences in habituation rate should be interpreted cautiously in light of their marginal significance and the small sample size. The NSSI group and psychiatric control group were matched on potentially confounding variables (e.g., psychopathology, suicide attempt history, childhood trauma, global functional impairment, demographic characteristics), suggesting that differences may 
specifically relate to aspects of prior engagement in NSSI. These findings highlight the importance of studying multiple aspects of the time course of responding to aversiveness (i.e., affective chronometry; Klumpp \& Shankman, 2018), and suggest that insensitivity to psychophysiological aversiveness may be a pathway through which NSSI history may lead to an escalation in NSSI and/or acquired capability for suicidal behavior.

The blunted initial reactivity in the NSSI group may have been due to prior habituation to aversiveness resulting from prior engagement in NSSI. This interpretation is consistent with the IPTS and gateway theory, as both theories suggest that prior aversive experiences (e.g., NSSI) cause habituation to aversiveness (Joiner, 2005; Smith \& Cukrowicz, 2010; Whitlock et al., 2013), which in turn reduces the defensive reaction or reflex to aversiveness (i.e., initial reactivity; Smith \& Cukrowicz, 2010) and increases acquired capability for suicide.

Although individuals with a history of NSSI did not significantly habituate, differences in habituation rates between the NSSI group and the two control groups were only marginally significant. It is therefore unclear whether NSSI history is only associated with blunted initial reactivity or whether it is also associated with blunted habituation to aversiveness, as it is possible that observed group differences in habituation rate were type I errors. Group differences in habituation rate should therefore be interpreted cautiously and are in need of replication. The NSSI group's marginally blunted rate of habituation relative to controls may appear to be in conflict with prior findings linking suicide attempts to faster electrodermal habituation (e.g., Edman et al., 1986; Jandl et al., 2010) and the IPTS's and gateway theory's assertion that exposure to NSSI should be associated with faster physiological habituation (Joiner, 2005; Whitlock et al., 2013). It is, however, consistent with a prior study that found that startle habituation was unrelated to suicide attempt history and acquired capability for suicide (Smith et al., 2015). Furthermore, considering that the NSSI group's initial reactivity was blunted relative to the two control groups and there were no group differences in startle response to the final startle probe, the NSSI group may have habituated to aversiveness before the start of the habituation task. In other words, individuals with a history of NSSI may have had less "far to fall" in terms of startle reactivity due to their blunted initial reactivity. Taken together, these results may suggest that individuals who have habituated to the aversiveness of NSSI may increase the frequency and/or severity of NSSI method to produce the desired experience of pain, which in turn might increase acquired capability for suicide.

Although it is unclear which specific aspects of NSSI our habituation task approximated, these findings provide insight into the role of responding to psychophysiological aversiveness in NSSI. Future studies are needed, however, to disambiguate whether blunted initial reactivity and slower habituation are vulnerability markers that precede NSSI or are a consequence (i.e., "scar") of NSSI. It is possible that blunted initial reactivity and slower habituation to aversiveness may be a trait like factor that precedes and connotes risk for NSSI. Alternatively, abnormal responding to aversiveness may be a consequence of exposure to the physiological aversiveness of NSSI (i.e., prior habituation). In line with this idea, preliminary longitudinal data suggest that pain sensitivity does not normalize after NSSI reduction (Koenig et al., 2017), but further longitudinal research is needed to elucidate the mechanistic role of responding to aversiveness in NSSI. 
This study had several notable strengths. First, we examined the time course of responding to aversiveness, which allowed us to differentiate initial reactivity and habituation. Several studies examining the association between average startle response and NSSI have reported no relationship between the affectively modulated startle reflex and history of NSSI (Franklin et al., 2013; Glenn et al., 2011). Our findings provide insight into the temporal dynamics of responding to aversiveness in individuals with a history of NSSI. Second, our examination of a transdiagnostic sample was important, as NSSI itself is transdiagnostic (Nock et al., 2006). Third, we included a psychiatric control group matched on potentially confounding variables (e.g., psychopathology, suicidality, childhood trauma, global functional impairment, demographics), which allowed us to show that blunted initial reactivity and slower habituation to aversiveness likely were not reflective of broader risk factors for suicide and may be specific to NSSI.

This study also had several limitations, the most notable of which relate to our utilization of data from a larger psychophysiological study that was not designed to examine differences in responding to aversiveness by NSSI history. First, our extracted groups (and the NSSI group in particular) were small, which likely decreased statistical power. Second, we used a single interview item to assess NSSI history. Single item assessments of NSSI, although commonly used, may yield lower estimates of NSSI prevalence than NSSI checklist measures (Muehlenkamp, Claes, Havertape, \& Plener, 2012) and may have greater potential for false negatives (i.e., participants incorrectly reporting no history of NSSI) and false positives (i.e., participants incorrectly reporting a history of NSSI; Ross \& Heath, 2002). However, the validity of our assessment of NSSI was supported by the fact that individuals with a history of NSSI exhibited a higher pain threshold compared to healthy controls and psychiatric controls, a finding that has been consistently reported in the NSSI literature (Koenig et al., 2016). Third, we did not assess the frequency or method of NSSI or time since last NSSI episode. Although several studies have found no relationship between NSSI frequency and responding to aversiveness (Glenn, Michel, Franklin, Hooley, \& Nock, 2014; Nock et al., 2006), it is possible that these variables may have affected our findings. Fourth, as previously discussed, it is unclear which elements of the NSSI process are most closely approximated by our startle habituation paradigm. Although similar paradigms have been used in other studies examining psychophysiological mechanisms of self-harm behaviors (Smith et al., 2015), acoustic startle probes may lack ecological validity as aversive stimuli (i.e., startle probes may not be subjectively aversive for those with a history of NSSI). It is also possible that habituation to reflexive defensive responding (measured via startle) could involve different mechanisms than habituation to intentional self-injury or other types of aversive stimuli. However, a variety of stimuli have been used as laboratory proxies for NSSI (e.g., heat pain, cold pain, shocks, imaginary scripts) and have yielded similar results (i.e., reduced physiological arousal or negative affect after the NSSI proxy; Franklin et al., 2010; Franklin et al., 2013; Haines, Williams, Brain, \& Wilson, 1995; Schmahl et al., 2006). Fifth, the omnibus group by time interaction and effect of group on pain threshold only approached statistical significance and thus follow up analyses examining specific group comparisons must be interpreted cautiously. Sixth, our extracted sample was entirely female. Although our findings indicate that insensitivity to aversiveness may be a mechanism 
through which NSSI leads to increased risk for suicidal behavior, there may be separate mechanisms for males.

\subsection{Conclusions}

In summary, our findings suggest that NSSI history is associated with insensitivity to aversiveness as indicated by blunted initial reactivity to aversiveness. The NSSI group's blunted initial defensive reactivity relative to both healthy controls and psychiatric controls indicates that the NSSI group's reduced initial response was not due to broader risk factors for NSSI or suicide and may have been specifically associated with aspects of prior NSSI exposure such as prior habituation to the psychophysiological aversiveness of NSSI. Blunted initial defensive reactivity in individuals with a history of NSSI may be a mechanism through which NSSI exposure leads to an escalation in NSSI (e.g., increased frequency or severity of NSSI method) and/or increased risk for suicidal behavior.

\section{Acknowledgements and Funding Sources}

This work was supported by a grant from the National Institute of Mental Health (R01MH098093 [PI: Shankman]). Other support for this work was provided by the University of Illinois at Chicago (UIC) Center for Clinical and Translational Science (CCTS) award number UL1RR029879 from the National Center for Research Resources.

\section{References}

American Psychiatric Association. (1994). Diagnostic and statistical manual of mental disorders: DSM-IV (4th ed.). Washington, DC: American Psychiatric Association.

Andover MS, \& Gibb BE (2010). Non-suicidal self-injury, attempted suicide, and suicidal intent among psychiatric inpatients. Psychiatry Research, 178(1), 101-105. 10.1016/j.psychres. 2010.03.019 [PubMed: 20444506]

Andover MS, Primack JM, Gibb BE, \& Pepper CM (2010). An examination of non-suicidal self-injury in men: do men differ from women in basic NSSI characteristics? Archives of Suicide Research, 14(1), 79-88. 10.1080/13811110903479086 [PubMed: 20112146]

Asarnow JR, Porta G, Spirito A, Emslie G, Clarke G, Wagner KD, Vitiello B, Keller M, Birmaher B, McCracken J, Mayes T, Berk M, ... Brent DA (2011). Suicide attempts and nonsuicidal self-injury in the treatment of resistant depression in adolescents: Findings from the TORDIA study. Journal of the American Academy of Child and Adolescent Psychiatry, 50(8), 772-781. 10.1016/j.jaac. 2011.04.003 [PubMed: 21784297]

Ballard ED, Ionescu DF, Vande Voort JL, Niciu MJ, Richards EM, Luckenbaugh DA, ... Zarate CA (2014). Improvement in suicidal ideation after ketamine infusion: Relationship to reductions in depression and anxiety. Journal of Psychiatric Research, 58, 161-166. 10.1016/j.psychires. 2014.07.027 [PubMed: 25169854]

Banks SJ, Eddy KT, Angstadt M, Nathan PJ, \& Phan KL (2007). Amygdala-frontal connectivity during emotion regulation. Social Cognitive and Affective Neuroscience, 2(4), 303-312. 10.1093/ scan/nsm029 [PubMed: 18985136]

Bates D, Maechler M, Bolker B, \& Walker S (2015). Fitting linear mixed-effects models using lme4. Journal of Statistical Software, 67(1), 1-48. 10.18637/jss.v067.i01.

Bernstein DP, Stein JA, Newcomb MD, Walker E, Pogge D, Ahluvalia T, ... Zule W. (2003). Development and validation of a brief screening version of the Childhood Trauma Questionnaire. Child Abuse \& Neglect, 27(2), 169-190. 10.1016/S0145-2134(02)00541-0

Blanch A, Balada F, \& Aluja A (2014). Habituation in acoustic startle reflex: Individual differences in personality. International Journal of Psychophysiology, 91(3), 232-239. 10.1016/j.ijpsycho. 2014.01.001 [PubMed: 24412342] 
Blumenthal TD, Cuthbert BN, Filion DL, Hackley S, Lipp OV, \& van Boxtel A (2005). Committee report: Guidelines for human startle eyeblink electromyographic studies. Psychophysiology, 42, 115. 10.1111/j.1469-8986.2005.00271.x [PubMed: 15720576]

Brackman EH, Morris BW, \& Andover MS (2016). Predicting risk for suicide: A preliminary examination of non-suicidal self-injury and the acquired capability construct in a college sample. Archives of suicide research, 20(4), 663-676. 10.1111/j.1469-8986.2005.00271.x [PubMed: 27007698]

Bresin K, \& Schoenleber M (2015). Gender differences in the prevalence of nonsuicidal self-injury: A meta-analysis. Clinical Psychology Review, 38, 55-64. 10.1016/J.CPR.2015.02.009 [PubMed: 25795294]

Campbell ML, Gorka SM, McGowan SK, Nelson BD, Sarapas C, Katz AC, Robison-Andrew EJ, ... Shankman SA (2013). Does anxiety sensitivity correlate with startle habituation? An examination in two independent samples. Cognition \& Emotion, 28(1), 46-58. 10.1080/02699931.2013.799062 [PubMed: 23746071]

Caspi A, Houts RM, Belsky DW, Goldman-Mellor SJ, Harrington H, Israel S, ... Moffitt TE (2014). The p factor: one general psychopathology factor in the structure of psychiatric disorders? Clinical Psychological Science, 2(2), 119-137. 10.1177/2167702613497473 [PubMed: 25360393]

Crawford J, Cayley C, Lovibond PF, Wilson PH, \& Hartley C (2011). Percentile norms and accompanying interval estimates from an Australian general adult population sample for selfreport mood scales (BAI, BDI, CRSD, CES-D, DASS, DASS-21, STAI-X, STAI-Y, SRDS, and SRAS). Australian Psychologist, 46(1), 3-14. 10.1111/j.1742-9544.2010.00003.x

Edman G, Åsberg M, Levander S, \& Schalling D (1986). Skin conductance habituation and cerebrospinal fluid 5-hydroxyindoleacetic acid in suicidal patients. Archives of General Psychiatry, 43(6), 586-592. 10.1001/archpsyc.1986.01800060080010 [PubMed: 2423049]

First M, Williams J, Karg R, \& Spitzer R (2015). Structured Clinical Interview for DSM-5: Research Version (SCID-5 for DSM-5, Research Version; SCID-5-RV).

Fleischmann A, Bertolote JM, Belfer M, \& Beautrais A (2005). Completed suicide and psychiatric diagnoses in young people: a critical examination of the evidence. American Journal of Orthopsychiatry, 75(4), 676-683. 10.1037/0002-9432.75.4.676 [PubMed: 16262523]

Fournier JC, DeRubeis RJ, Hollon SD, Dimidjian S, Amsterdam JD, Shelton RC, \& Fawcett J (2010). Antidepressant drug effects and depression severity: A patient-level meta-analysis. Journal of the American Medical Association, 303(1), 47-53. 10.1001/jama.2009.1943 [PubMed: 20051569]

Franklin JC, Hessel ET, Aaron RV, Arthur MS, Heibron N, \& Prinstein MJ (2010). The functions of nonsuicidal self-injury: Support for cognitive-affective regulation and opponent processes from a novel psychophysiological paradigm. Journal of Abnormal Psychology, 119(4), 850-862. 10.1037/ a0020896 [PubMed: 20939652]

Franklin JC, Lee KM, Hanna EK, \& Prinstein MJ (2013). Feeling worse to feel better: Pain-offset relief simultaneously stimulates positive affect and reduces negative affect. Psychological Science, 24(4), 521-529. 10.1177/0956797612458805 [PubMed: 23459871]

Franklin JC, Puzia ME, Lee KM, Lee GE, Hanna EK, Spring VL, \& Prinstein MJ (2013). The nature of pain offset relief in nonsuicidal self-injury: A laboratory study. Clinical Psychological Science, 1(2), 110-119. 10.1177/2167702612474440

Glassman LH, Weierich MR, Hooley JM, Deliberto TL, \& Nock MK (2007). Child maltreatment, nonsuicidal self-injury, and the mediating role of self-criticism. Behaviour Research and Therapy, 45(10), 2483-2490. 10.1016/j.brat.2007.04.002 [PubMed: 17531192]

Glenn CR, Blumenthal TD, Klonsky ED, \& Hajcak G (2011). Emotional reactivity in nonsuicidal selfinjury: Divergence between self-report and startle measures. International Journal of Psychophysiology, 80(2), 166-170. 10.1016/j.ijpsycho.2011.02.016 [PubMed: 21376761]

Glenn CR, \& Klonsky ED (2013). Nonsuicidal self-injury disorder: An empirical investigation in adolescent psychiatric patients. Journal of Clinical Child and Adolescent Psychology, 42(4), 496507. 10.1080/15374416.2013.794699 [PubMed: 23682597]

Glenn JJ, Michel BD, Franklin JC, Hooley JM, \& Nock MK (2014). Pain analgesia among adolescent self-injurers. Psychiatry Research, 220(3), 921-926. 10.1016/j.psychres.2014.08.016 [PubMed: 25172611] 
Goldman HH, Skodol AE, \& Lave TR (1992). Revising Axis V for DSM-IV: A review of measures of social functioning. American Journal of Psychiatry, 149(9), 1148-1156. 10.1176/ajp.149.9.1148 [PubMed: 1386964]

Gorka SM, Hee D, Lieberman L, Mittal VA, Phan KL, \& Shankman SA (2016). Reactivity to uncertain threat as a familial vulnerability factor for alcohol use disorder. Psychological Medicine, 46(16), 3349-3358. 10.1017/S0033291716002415 [PubMed: 27624067]

Gorka SM, Liu H, Sarapas C, \& Shankman SA (2015). Time course of threat responding in panic disorder and depression. International Journal of Psychophysiology, 98 (1), 87-94. 10.1016/ j.ijpsycho.2015.07.005 [PubMed: 26168883]

Gorka SM, Nelson BD, \& Shankman SA (2013). Startle response to unpredictable threat in comorbid panic disorder and alcohol dependence. Drug and Alcohol Dependence, 132(1-2), 216-222. 10.1016/j.drugalcdep.2013.02.003 [PubMed: 23465734]

Guan K, Fox KR, \& Prinstein MJ (2012). Nonsuicidal self-injury as a time-invariant predictor of adolescent suicide ideation and attempts in a diverse community sample. Journal of Consulting and Clinical Psychology, 80(5), 842-849. 10.1037/a0029429 [PubMed: 22845782]

Haines J, Williams CL, Brain KL, \& Wilson GV (1995). The psychophysiology of self-mutilation. Journal of Abnormal Psychology, 104, 471-489. [PubMed: 7673571]

Hamza CA, Stewart SL, \& Willoughby T (2012). Examining the link between nonsuicidal self-injury and suicidal behavior: A review of the literature and an integrated model. Clinical Psychology Review, 32(6), 482-495. 10.1016/J.CPR.2012.05.003 [PubMed: 22717336]

Heath NL, Toste JR, Nedecheva T, \& Charlebois A (2008). An examination of nonsuicidal self-injury among college students. Journal of Mental Health Counseling, 30, 137-156. 10.17744/mehc. 30.2.8p879p3443514678

Jacobson CM, \& Gould M (2007). The epidemiology and phenomenology of non-suicidal selfinjurious behavior among adolescents: A critical review of the literature. Archives of Suicide Research, 11(2), 129-147. 10.1080/13811110701247602 [PubMed: 17453692]

Jaeger B (2017). r2glmm: Computes R Squaredfor Mixed (Multilevel) Models. R package version 0.1.2. https://CRAN.R-proiect.org/package=r2glmm

Jandl M, Steyer J, \& Kaschka WP (2010). Suicide risk markers in major depressive disorder: A study of electrodermal activity and event-related potentials. Journal of Affective Disorders, 123(1-3), 138-149. 10.1016/JJAD.2009.09.011 [PubMed: 19819558]

Joiner TE (2005). Why People Die by Suicide. Cambridge, MA: Harvard University Press.

Jovanovic T, \& Ressler KJ (2010). How the neurocircuitry and genetics of fear inhibition may inform our understanding of PTSD. American Journal of Psychiatry, 167(6), 648-662. 10.1176/appi.ajp. 2009.09071074 [PubMed: 20231322]

Keller F, Wolfersdorf M, Straub R, \& Hole G (1991). Suicidal behaviour and electrodermal activity in depressive inpatients. Acta Psychiatrica Scandinavica, 83(5), 324-328. 10.1111/j. 1600-0447.1991.tb05549.x [PubMed: 1853722]

Klonsky ED (2011). Non-suicidal self-injury in United States adults: Prevalence, sociodemographics, topography, and functions. Psychological Medicine, 41, 1981-1986. 10.1017/S0033291710002497 [PubMed: 21208494]

Klonsky ED (2007). The functions of deliberate self-injury: A review of the evidence. Clinical Psychology Review, 27(2), 226-239. 10.1016/j.cpr.2006.08.002 [PubMed: 17014942]

Klonsky ED (2018). Non-suicidal self-injury in United States adults: Prevalence, sociodemographics, topography and functions. Psychological Medicine, 41(9), 1981-1986. 10.1017/ S0033291710002497

Klumpp H, \& Shankman SA (2018). Using event-related potentials and startle to evaluate time course in anxiety and depression. Biological Psychiatry: Cognitive Neuroscience and Neuroimaging, 3, 10-18. 10.1016/j.bpsc.2017.09.004 [PubMed: 29397073]

Koenig J, Rinnewitz L, Niederbäumer M, Strozyk T, Parzer P, Resch F, \& Kaess M (2017). Longitudinal development of pain sensitivity in adolescent non-suicidal selfinjury. Journal of Psychiatric Research, 89, 81-84. 10.1016/j.psychires.2017.02.001 [PubMed: 28193582]

Koenig J, Thayer JF, \& Kaess M (2016). A meta-analysis on pain sensitivity in self-injury. Psychological Medicine, 46(8), 1597-1612. 10.1017/S0033291716000301 [PubMed: 26964517] 
Kotov R, Chang S-W, Fochtmann LJ, Mojtabai R, Carlson GA, Sedler MJ, \& Bromet EJ (2011). Schizophrenia in the intemalizing-extemalizing framework: A third dimension? Schizophrenia Bulletin, 37(6), 1168-1178. 10.1093/schbul/sbq024 [PubMed: 20357134]

Krueger RF, Caspi A, Moffitt TE, \& Silva PA (1998). The structure and stability of common mental disorders (DSM-III-R): A longitudinal-epidemiological study. Journal of Abnormal Psychology, 107(2), 216-227. 10.1037/0021-843X.107.2.216 [PubMed: 9604551]

Kuznetsova A, Brockhoff PB, \& Christensen RHB (2017). ImerTest package: Tests in linear mixed effects models. Journal of Statistical Software, 82(13), 1-26. 10.18637/jss.v082.i13

Lang PJ (1995). The emotion probe: Studies of motivation and attention. American Psychologist, 50(5), 372-385. [PubMed: 7762889]

Lovibond S, \& Lovibond P (1995). Manual for the depression anxiety stress scales Sydney, Australia: The Psychology Foundation of Australia, Inc.

Luke SG (2017). Evaluating significance in linear mixed-effects models in R. Behavior Research Methods, 49(A), 1494-1502. 10.3758/s13428-016-0809-y [PubMed: 27620283]

Markon KE (2010). Modeling psychopathology structure: A symptom-level analysis of axis I and II disorders. Psychological Medicine, 40(2), 273 10.1017/S0033291709990183 [PubMed: 19515267]

Miller GA, \& Chapman JP (2001). Misunderstanding analysis of covariance. Journal of Abnormal Psychology, 110(1), 40-48. 10.1037/0021-843X.110.T40 [PubMed: 11261398]

Miller MW, Curtin JJ, \& Patrick CJ (1999). A startle-probe methodology for investigating the effects of active avoidance on negative emotional reactivity. Biological Psychology, 50(3), 235-257. 10.1016/S0301-0511(99)00011-3 [PubMed: 10461807]

Muehlenkamp JJ, Claes L, Havertape L, \& Plener PL (2012). International prevalence of adolescent non-suicidal self-injury and deliberate self-harm. Child and Adolescent Psychiatry and Mental Health, 6 (10). 10.1186/1753-2000-6-10

Muehlenkamp JJ, \& Gutierrez PM (2007). Risk for suicide attempts among adolescents who engage in non-suicidal self-injury. Archives of Suicide Research, 11(1), 69-82. 10.1080/13811110600992902 [PubMed: 17178643]

Nock MK (2009). Why do People Hurt Themselves? New Insights Into the Nature and Functions of Self-Injury. Current Directions in Psychological Science, 18(2), 78-83. 10.1111/j. 1467-8721.2009.01613.x [PubMed: 20161092]

Nock MK, Joiner TE, Gordon KH, Lloyd-Richardson E, \& Prinstein MJ (2006). Non-suicidal selfinjury among adolescents: Diagnostic correlates and relation to suicide attempts. Psychiatry Research, 144(1), 65-72. 10.1016/j.psychres.2006.05.010. [PubMed: 16887199]

Nock MK, \& Mendes WB (2008). Physiological arousal, distress tolerance, and social problemsolving deficits among adolescent self-injurers. Journal of Consulting and Clinical Psychology, 76(1), 28-38. 10.1037/0022-006X.76.1.28 [PubMed: 18229980]

Phan KL, Fitzgerald DA, Nathan PJ, Moore GJ, Uhde TW, \& Tancer ME (2005). Neural substrates for voluntary suppression of negative affect: A functional magnetic resonance imaging study. Biological Psychiatry, 57(3), 210-219. 10.1016/j.biopsych.2004.10.030 [PubMed: 15691521]

Prinstein MJ, Nock MK, Simon V, Aikins JW, Cheah CSL, \& Spirito A (2008). Longitudinal trajectories and predictors of adolescent suicidal ideation and attempts following inpatient hospitalization. Journal of Consulting and Clinical Psychology, 76(1), 92-103. 10.1037/0022-006X.76.1.92 [PubMed: 18229987]

R Core Team (2017). R: A language and environment for statistical computing R Foundation for Statistical Computing. Vienna, Austria https://www.R-project.org/

Rodriguez-Fornells A, Riba J, Gironell A, Kulisevsky J, \& Barbanoj MJ (1999). Effects of alprazolam on the acoustic startle response in humans. Psychopharmacology, 143(3), 280-285. 10.1007/ s002130050948 [PubMed: 10353431]

Rollman GB, \& Harris G (1987). The detectability, discriminability, and perceived magnitude of painful electrical shock. Perception \& Psychophysics, 42(3), 257-268. 10.3758/BF03203077 [PubMed: 3671051]

Ross S \& Heath N (2002). A study of the frequency of self-mutilation in a community sample of adolescents. Journal of Youth and Adolescence, 31(1), 6777 10.1023/A:1014089117419 
Schmahl C, Bohus M, Esposito F, Rolf-Detlef T, Di Salle F, Greffrath W, Ludaescher A, ... Seifritz E (2006). Neural correlates of antinociception in borderline personality disorder. Archives of General Psychiatry, 63, 659-667. 10.1001/archpsyc.63.6.659 [PubMed: 16754839]

Shankman SA, Funkhouser CJ, Klein DN, Davila J, Lemer D, \& Hee D (2018). Reliability and validity of severity dimensions of psychopathology assessed using the Structured Clinical Interview for DSM-5 (SCID). International Journal of Methods in Psychiatric Research, 27(1). 10.1002/mpr. 1590

Shankman SA, Nelson BD, Sarapas C, Robison-Andrew EJ, Campbell ML, Altman SE, ... Gorka SM (2013). A psychophysiological investigation of threat and reward sensitivity in individuals with panic disorder and/or major depressive disorder. Journal of Abnormal Psychology, 122(2), 322338. 10.1037/a0030747 [PubMed: 23148783]

Shrout PE (1998). Measurement reliability and agreement in psychiatry. Statistical Methods in Medical Research, 7(3), 301-317. 10.1191/096228098672090967 [PubMed: 9803527]

Singmann H, Bolker B, Westfall J, \& Aust F (2017). afex: Analysis of Factorial Experiments. R package version 0.18-0. https://CRAN.R-project.org/package=afex

Smith PN, \& Cukrowicz KC (2010). Capable of suicide: A functional model of the acquired capability component of the interpersonal-psychological theory of suicide. Suicide and Life-Threatening Behavior, 40(3), 266-275. 10.1521/suli.2010.40.3.266 [PubMed: 20560748]

Smith PN, Wolford-Clevenger C, Selwyn CN, Poindexter E, Lechner W, Grant DM, \& Cukrowicz KC (2015). An exploratory analysis of the relations between the rate of physiological habituation, the acquired capability for suicide, and acute risk factors for suicide. Journal of Aggression, Conflict and Peace Research, 7(3), 139-148. 10.1108/jacpr-07-2014-0130

Sornberger MJ, Heath NL, Toste JR, \& McLouth R (2012). Nonsuicidal self-injury and gender: Patterns of prevalence, methods, and locations among adolescents. Suicide and Life-Threatening Behavior, 72(3), 266-278. 10.1111/j.1943-278X.2012.0088.x

Thorell LH, Wolfersdorf M, Straub R, Steyer J, Hodgkinson S, Kaschka WP, \& Jandl M (2013). Electrodermal hyporeactivity as a trait marker for suicidal propensity in uni- and bipolar depression. Journal of Psychiatric Research, 77(12), 1925-1931. 10.1016/j.jpsychires.2013.08.017

Victor SE, \& Klonsky ED (2014). Correlates of suicide attempts among self-injurers: A meta-analysis. Clinical Psychology Review, 34(4), 282-297. 10.1016/j.cpr.2014.03.005 [PubMed: 24742496]

Victor SE, Muehlenkamp JJ, Hayes NA, Lengel GJ, Styer DM, \& Washburn JJ (2018). Characterizing gender differences in nonsuicidal self-injury: Evidence from a large clinical sample of adolescents and adults. Comprehensive Psychiatry, 82, 53-60. 10.1016/j.comppsych.2018.01.009 [PubMed: 29407359]

Westlund Schreiner M, Klimes-Dougan B, Begnel ED, \& Cullen KR (2015). Conceptualizing the neurobiology of non-suicidal self-injury from the perspective of the Research Domain Criteria Project. Neuroscience \& Biobehavioral Reviews, 57, 381-391. 10.1016/j.neubiorev.2015.09.011

Whitlock J, Muehlenkamp J, Eckenrode J, Purington A, Baral Abrams G, Barreira P, \& Kress V (2013). Nonsuicidal self-injury as a gateway to suicide in young adults. Journal of Adolescent Health, 52(4), 486-492. 10.1016/j.jadohealth.2012.09.010 [PubMed: 23298982]

Wilkinson P, Kelvin R, Roberts C, Dubicka B, \& Goodyer I (2011). Clinical and psychosocial predictors of suicide attempts and nonsuicidal self-injury in the Adolescent Depression Antidepressants and Psychotherapy Trial (ADAPT). American Journal of Psychiatry, 168(5), 495501. 10.1176/appi.ajp.2010.10050718 [PubMed: 21285141]

Wolfersdorf M, Straub R, Barg T, Keller F, \& Kaschka WP (1999). Depressed inpatients, electrodermal reactivity, and suicide - a study about psychophysiology of suicidal behavior. Archives of Suicide Research, 5(1), 1-10. 10.1080/13811119908258311 


\section{Highlights}

- $\quad$ NSSI history was associated with blunted initial reactivity to aversiveness

- $\quad$ This effect was not due to co-occurring suicide risk factors (e.g. psychopathology)

- $\quad$ Those with a history of NSSI habituated marginally slower than healthy controls

- Initial insensitivity to aversiveness may increase risk for suicidal behavior 


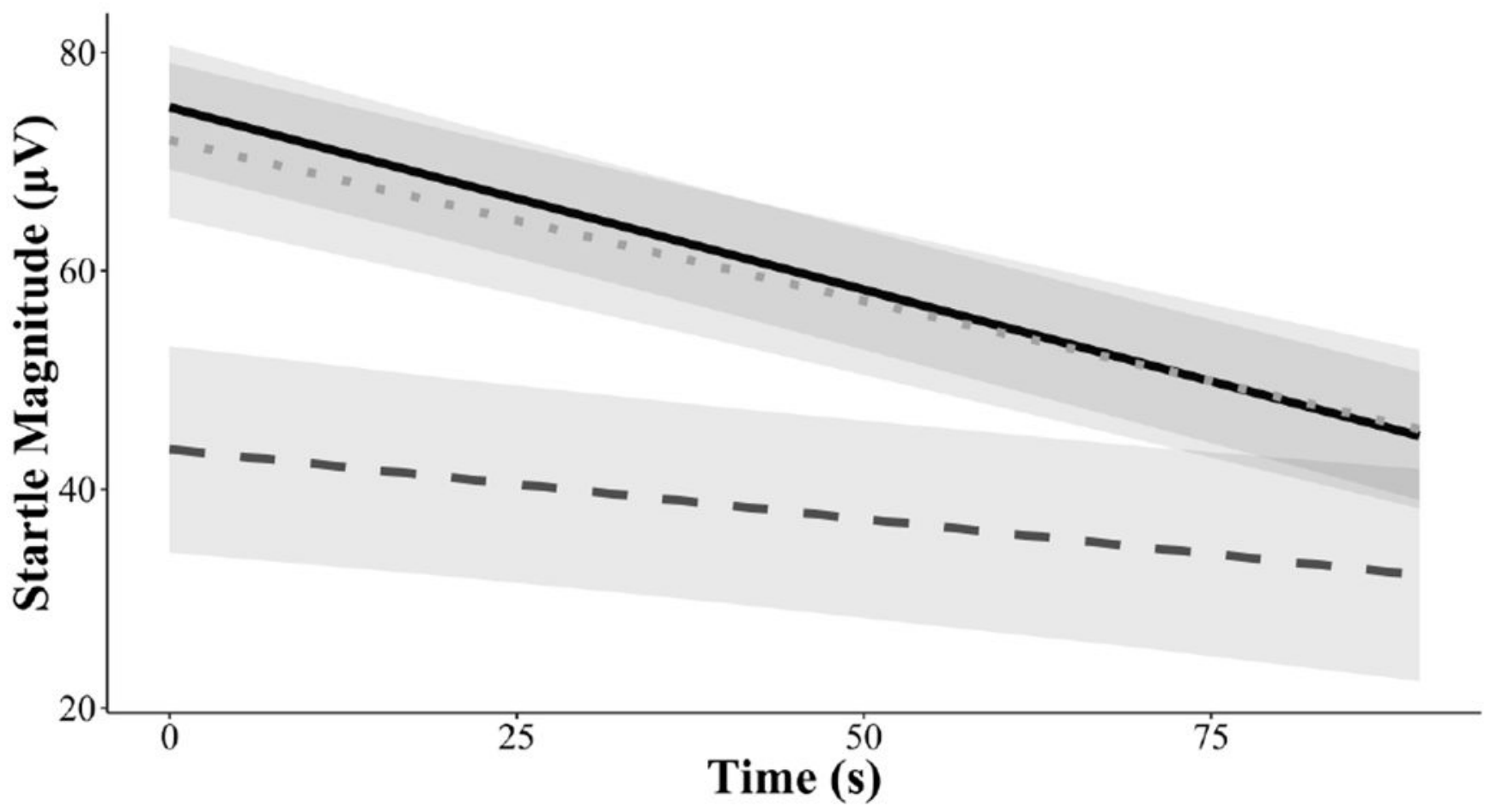

-Healthy Controls=NSSI" Psychiatric Controls

Figure 1.

Group differences in the time course of startle responding. Ribbons represent standard errors. 
Table 1.

Participant Demographics and Clinical Characteristics

\begin{tabular}{|c|c|c|c|c|}
\hline & NSSI group $(n=16)$ & $\begin{array}{l}\text { Psychiatric Controls }(n= \\
\text { 28) }\end{array}$ & $\begin{array}{l}\text { Healthy Controls }(n= \\
\text { 43) }\end{array}$ & $\begin{array}{l}p \text {-value of group } \\
\text { difference test }\end{array}$ \\
\hline $\operatorname{Age}(M, S D)$ & $22.19(2.88)$ & $21.11(1.89)$ & $21.63(3.01)$ & .429 \\
\hline Ethnicity (\% Caucasian) & $62.5 \%$ & $32.1 \%$ & $48.8 \%$ & .132 \\
\hline Years of Education $(M, S D)$ & $14.87(1.97)$ & $14.92(1.74)$ & $15.51(1.98)$ & .346 \\
\hline Current psychiatric medication & $31.3 \%$ & $25.0 \%$ & - & .924 \\
\hline Lifetime suicide attempt & $12.5 \%$ & $17.9 \%$ & - & .969 \\
\hline Lifetime suicidal ideation & $75.0 \%$ & $46.4 \%$ & - & .128 \\
\hline Current suicidal ideation & $6.25 \%$ & $10.7 \%$ & - & .999 \\
\hline Lifetime MDD & $93.8 \%$ & $85.7 \%$ & - & .753 \\
\hline Current MDD & $18.8 \%$ & $25.0 \%$ & - & .919 \\
\hline Lifetime AUD or SUD & $62.5 \%$ & $57.1 \%$ & - & .977 \\
\hline Current AUD or SUD & $6.3 \%$ & $17.9 \%$ & - & .534 \\
\hline Lifetime PD & $37.5 \%$ & $39.3 \%$ & - & .999 \\
\hline Current PD & $18.8 \%$ & $14.3 \%$ & - & .999 \\
\hline Lifetime GAD & $26.7 \%$ & $28.6 \%$ & - & .999 \\
\hline Current GAD & $18.8 \%$ & $7.1 \%$ & - & .501 \\
\hline $\operatorname{GAF}(M, S D)$ & $62.50(12.92)^{\mathrm{a}}$ & $60.46(11.40)^{\mathrm{a}}$ & $84.00(8.57)^{\mathrm{b}}$ & $<.001$ \\
\hline SOFAS $(M, S D)$ & $67.19(11.78)^{\mathrm{a}}$ & $65.18(12.59)^{\mathrm{a}}$ & $85.44(7.45)^{\mathrm{b}}$ & $<.001$ \\
\hline CTQ Physical Abuse $(M, S D)$ & $7.00(2.94)^{\mathrm{a}, \mathrm{b}}$ & $7.18(2.28)^{\mathrm{a}}$ & $5.93(2.19)^{\mathrm{b}}$ & .070 \\
\hline CTQ Sexual Abuse $(M, S D)$ & $7.19(5.68)^{\mathrm{a}}$ & $5.96(2.62)^{\mathrm{a}, \mathrm{b}}$ & $5.21(.68)^{\mathrm{b}}$ & .064 \\
\hline CTQ Emotional Abuse $(M, S D)$ & $9.75(4.82)^{\mathrm{a}}$ & $9.68(3.51)^{\mathrm{a}}$ & $6.95(3.02)^{\mathrm{b}}$ & .002 \\
\hline CTQ Physical Neglect $(M, S D)$ & $7.06(2.54)^{\mathrm{a}, \mathrm{b}}$ & $7.43(2.67)^{\mathrm{a}}$ & $5.91(2.23)^{\mathrm{b}}$ & .031 \\
\hline CTQ Emotional Neglect $(M, S D)$ & $10.81(4.83)^{\mathrm{a}}$ & $9.61(3.89)^{\mathrm{a}}$ & $7.00(2.65)^{\mathrm{b}}$ & $<.001$ \\
\hline
\end{tabular}

$\mathrm{MDD}=$ Major Depressive Disorder. AUD = Alcohol Use Disorder. SUD = Substance Use Disorder. PD = Panic Disorder. GAD = Generalized Anxiety Disorder. GAF = Global Assessment of Functioning. SOFAS = Social and Occupational Functioning Assessment Scale. CTQ = Childhood Trauma Scale-Short Form. Different subscripts within the same row reflect group differences $(p<.05)$. $p$-values of group difference tests for rows in which the healthy control group has a hyphen reflect comparisons between the NSSI and psychiatric control groups (i.e., excluding the healthy control group). Group differences for categorical variables were tested using chi-square tests with Yates' continuity correction. Group differences for continuous variables were tested using ANOVAs. 